\title{
THE THEORIES ON THE FORMATION OF THE AQUEOUS HUMOUR*
}

\author{
BY \\ J. Douglas Robertson, M.D. \\ LONDON \\ (FROM THE COURTAULD INSTITUTE OF BIOCHEMISTRY, \\ MIDDLESEX HOSPITAL, W.1)
}

For many years there has been controversy, becoming very bitter at times, regarding the mechanism of formation of the aqueous humour. This fluid has at various times been considered a dialysate, a transudate, an exudate or a secretion, and very strong arguments have been brought forward for and against the various concepts by particular experimentalists partial to one or the other theory.

Before going on to a study of the various theories in relation to the eye, it is important to define what is meant by the terms dialysate and ultrafiltrate, exudate, transudate, and secretion. As an introduction to their definitions I would like to refer very briefly to crystalloids and colloids, and to the permeability of membranes.

Crystalloids and Colloids.-The division of substances into the two large groups of crystalloids and colloids is due to the work of Graham (1876) the pioneer in colloidal research. Crystalloids, he maintained, examples of which are sodium chloride, sugar and magnesium sulphate, are fast diffusers, whereas colloids, examples of which are albumen, caramel, silicic acid, gum-arabic and tannin, are slow diffusers. As a general rule when solutions of colloids are evaporated to dryness they leave a gluey or glassy residue in contrast to crystalloids that crystallise out in saturated solutions. There are, however, certain colloids capable of crystallisation such as vegetable proteins, haemoglobin, and egg and serum albumen, so that crystalloids in contrast to colloids might be simply defined as substances with a high rate of diffusion and crystallising out easily in saturated solution.

The permeability of membranes.-A membrane may be completely impermeable. This is very rare but it occurs in the case of the membrane surrounding an egg. Most membranes are permeable to a small or large degree, depending on the fineness of the grain-i.e., the size of the holes through which the fluid passes. Filter paper permits the passage of water and all dissolved substances crystalloid or colloid. Gel membranes such as gelatin collodion or cellophane are of porous structure and they

-Lecture delivered at the Royal London Ophthalmic Hospital on November 25. 1938. 
have varying degrees of permeability. Some gel membranes are of coarse texture and they allow the passage of colloids as well as crystalloids, on the other hand some like cellophane keep back all the colloids and only allow the passage of water and crystalloids.

In general terms it may be said that the ability to pass through a membrane depends on the size of the particle in solution, and a semi-permeable membrane, for example cellophane, acts like a filter paper made infinitely fine.

Dialysates and ultrafiltrates.-Ultrafiltration may be defined as : " the process of separating substances in solution from one another." Dialysis is therefore a special type of ultrafiltration the process of which consists in separating crystalloids from colloids. The process of dialysis can be explained by taking a very simple example where this occurs. If we put some fluid containing a mixture of colloids and crystalloids (e.g., blood plasma) in a bag of cellophane and hang it up in a large tank of distilled water, the crystalloids such as urea, sugar, etc., will diffuse out through the cellophane membrane into the tank. If the water in the tank is changed frequently, then eventually we obtain a fluid inside the cellophane membrane quite free from salts and crystalloids but containing the whole of the colloids originally present. The serum will of course be diluted with water because the colloids retained inside the cellophane possess the property of attracting fluid. This process of freeing a colloidal solution from crystalloids is known as dialysis.

Osmotic Pressure.-The property which indiffusible colloids have in attracting fluid through a membrane is known as their osmotic pressure. 'The osmotic pressure of colloid varies according to the amount of colloid present.

Thus 1 gram per 100 c.c. of albumen attracts $7.54 \mathrm{~cm}$. water. 10 gram per 100 c.c. of albumen would attract $75.4 \mathrm{~cm}$. water. 100 c.c. blood plasma which is a mixture of albumen and g!obulin attracts $40 \mathrm{~cm}$. water.

Production of a pure ultrafiltrate of blood plasma.-If instead of placing the cellophane bag containing blood plasma in a tank of water, a certain pressure is put on the contents of the cellophane bag then fluid will pass out of the bag by so-called ultrafiltration. If the pressure is over $40 \mathrm{~cm}$. water (or greater than the osmotic pressure of the colloids in the serum) then a filtrate will be produced through the cellophane membrane. This we call an ultrafiltrate or a dialysate of blood.

So when we speak of a biological fluid as being a dialysate or ultrafiltrate of blood it means the production or formation of that fluid is similar to that which has just been described. Instead of the cellophane membrane we have the simple endothelial layers 
found in the blood and lymph capillaries, glomerulus of the kidney or on serous surfaces. From our knowledge of physical chemistry we know that water and non-colloidal solutes will endeavour to flow across the capillary membrane always to the side which contains protein or colloids in greater concentration. Some force is required to balance the osmotic pressure of the colloids in such systems. This force is found in the hydrostatic pressure. In certain parts of the capillary the hydrostatic or blood pressure in the capillary exceeds the colloid osmotic pressure by an amount sufficient to produce an ultrafiltration and so we have the formation of an ultrafiltrate of blood produced exactly as we would manufacture it experimentally.

What are the characteristics of dialysates or ultrafiltrates? They may be divided into physical and chemical.

Physical.-The physical equilibrium is such that the formation of dialysates in the body is governed by simple physical laws reproducible in vitro. 'The hydrostatic or intracapillary blood pressure wants to drive filterable fluid through the membrane, whereas the indiffusible colloids want to attract fluid inside the capillary. The circulation of fluid to and fro over the capillary membrane, which we know to exist, is explained in two ways.

a. At the arterial end of the capillary the hydrostatic pressure is greater than colloid osmotic pressure, whereas at the venous end of the capillary, the hydrostatic pressure is less than the colloid osmotic pressure. By this arrangement, tissue fluid is produced at the arterial end and absorbed at the venous end. It thus allows of a circulation of fluid through the tissues.

b. Against this simple mechanism it has been suggested that the capillaries throughout their entire length are either producing filtrate or absorbing fluid, so the hydrostatic capillary pressure at one time exceeds the colloid osmotic pressure and at another time is less than it.

The pressure of the filtrate or the tissue fluid pressure must obviously depend upon the hydrostatic pressure and the colloid osmotic pressure and the relationship is as follows. The dialysate pressure $=$ the hydrostatic pressure in the capillaries, minus the difference in osmotic pressure between blood and the dialysate.

Now the difference in osmotic pressure between blood and its dialysates is simply the difference in osmotic pressure of the fraction of indiffusible protein because there is complete equilibrium otherwise. So we may rewrite this equation : the dialysate pressure $=$ hydrostatic pressure - difference in colloid osmotic pressure of blood and the dialysate. Thus if the dialysate contains no protein at all, the dialysate pressure $=$ hydrostatic pressure minus the colloid osmotic pressure of the plasma.

The Chemical Equilibrium.-The question of the chemical 
equilibrium was fully discussed in my second lecture, but to summarise the various points :-

1. Protein may be present in varying amounts depending on the permeability of the capillary membrane.

2. Freely diffusible non-electrolytes such as non-protein nitrogen, urea, creatinine, uric acid, and sugar are present, in equal concentrations in blood plasma and its dialysates.

3. Cations such as sodium, potassium, magnesium, and calcium are present in greater concentration in the blood plasma than in the dialysate.

4. Anions such as chloride and phosphate, are present in less concentration in the blood than in the dialysate.

5 . The product of any pair of diffusible cations and anions in the blood is equal to the product of the same pair of cations and anions on the other side.

Thus-

$[\mathrm{Na}]$ blood $\times[\mathrm{Cl}]$ blood $=[\mathrm{Na}]$ dialysate $\times[\mathrm{Cl}]$ dialysate where the concentrations of $\mathrm{Na}$ and $\mathrm{Cl}$ are expressed in grams molecules per litre of solutions.

Regarding dialysates of blood therefore, there is definite physicochemical equilibrium, and for a fluid to be termed a dialysate it must conform to this equilibrium.

Exudate and transudate.-These filtrates of blood can be conveniently studied together. In dialysis the formation of fluid is governed by a simple physical process associated with an exchange of fluid over a membrane. It is possible that the permeability of this membrane may be altered by influences such as anoxaemia, bacterial or chemical toxins, so that the normal interchange of fluid becomes modified in the direction of increased accumulation of fluid. We meet with this clinically in oedema and in accumulation of fluid in the pleura, pericardium, and peritoneum. If the process is non-inflammatory in origin the product is called a transudate. If the process is inflammatory in origin the product is called an exudate.

It is often very difficult indeed to differentiate one from the other, but the following means have been adopted with a certain measure of success.

1. The specific gravity of transudates is below 1,015 and of exudates above 1,018 .

2. Exudates contain over 3 per cent. of protein, and transudates under 3 per cent.

3. Transudates are sterile, exudates may contain specific organisms.

4. Exudates contain polymorphonuclear cells in acute infections, and lymphocytes in chronic infections, whereas transudates contain few, if any cells. 
The physical and the chemical equilibria of transudates and exudates do not differ from those of dialysates.

Secretion.-One may define secretion in several ways and the following have been suggested.

1. Secretion is the elaboration of a product which contains some substance not present in the mother liquor, or present in marked excess in comparison with the mother liquor.

2 . Secretion is the elaboration of a product whose formation is the result of work done by the tissues.

There is no doubt that secretions in the body are derived from the plasma by some complex mechanism ill-understood. The control of secretion has variously been ascribed to a nervous, hormonal, and/or electrolytic mechanism.

Now let us apply these various definitions to the fluid produced in the eye.

Is the aqueous humour a dialysate?-If the aqueous humour is a dialysate, then-

1. The dialysing membrane must be the capillary wall, and thus fluid interchange must occur in both directions over the capillary membrane throughout the eye as a whole.

2 . The aqueous must resemble tissue fluid produced elsewhere in the body.

3. The physical equilibrium is such that the intra-ocular pressure must equal the hydrostatic pressure in the capillaries minus the difference in the osmotic pressure of the blood and aqueous humour. In other words it means that the intra-ocular pressure should vary with alterations in the osmotic pressure of blood in the same way as tissue fluid pressure does.

4. The chemical equilibrium should be similar to that of an ultrafiltrate of blood in vitro.

Let us deal with these various points.

1. The dialysate membrane.- It has been shown that the bloodaqueous barrier in the posterior chamber allows the free passage of water and dissolved substances from the blood to the aqueous. But when the permeability of the ciliary epithelium was tried in the opposite direction, a different result was obtained. Neither water nor dissolved substances was found to permeate the membrane passing in the direction from aqueous to blood. This is an important finding for it means the membrane in the posterior chamber of the eye has an irreversible permeability and therefore the passage of fluid across its walls must be accomplished at the expense of work done by the tissues. It is therefore incorrect to assume, as upholders of dialysis do, that fluid interchange occurs in both directions throughout the eye as a whole.

2 . The physical equilibrium in the eye cannot be explained on the simple laws governing dialysis. 
a. When the colloid osmotic pressure of the plasma falls below $15 \mathrm{~mm} . \mathrm{Hg}$ and nephrotic oedema or increased formation of the tissue fluid occurs, no change takes place in the level of the intraocular pressure.

$b$. Dehydration of the tissues with 50 per cent. glucose does not affect the intra-ocular pressure.

c. Dehydration of the tissues with 30 per cent. $\mathrm{NaCl}$ is at its maximum at the end of the injection and $\frac{3}{4}$ hour later the fluid content of the tissues is normal.

One hour after the injection of $\mathrm{NaCl}$, when the water content of the tissues is normal, the intra-ocular pressure is at a minimum.

3 . The chemical composition of the aqueous humour is not comparable to that of an ultrafiltrate of plasma.

a. Freely diffusible and easily filterable substances such as urea, uric acid, glucose, and creatinine, do not readily diffuse into the aqueous humour. These substances actually pass over the secreting membrane of the stomach more easily-Robertson and Williams (1939).

$b$. With the exception of $\mathrm{Na}$ and $\mathrm{Cl}$ in the opinion of most workers the distributions of ions in the aqueous humour is not in accord with that one would expect from dialysis-Robertson (1939).

4. The aqueous humour therefore, does not resemble lymph which is an example of a fluid produced by dialysis.

Our conclusions are therefore, that the aqueous humour does not fulfil the necessary conditions for it to be considered an ultrafiltrate or dialysate of blood.

Is the aqueous humour a transudate?-I do not think, on the evidence we have, that the aqueous can be called a transudate. It is not an exudate for there is no process of inflammation, which according to the definition of the word, is necessary.

The physical and chemical equilibrium of transudates does not appear to differ in any way from that of dialysates. I cannot understand, therefore, an objection that has been raised against the transudation theory by supporters of the dialysis theorynamely, that the aqueous humour has more chlorides than the plasma. So far as I know all transudates have chlorides in excess of the blood plasma. Further, it has been stated in criticism of the transudation theory- "If the aqueous humour is formed by a pressure transudation and circulates actively through the eye and if it is not a product of thermo-dynamical equilibrium, then the one fact that it contains more chloride ions than plasma forces one to postulate that it is formed by a gland secreting saline." This of course, is not so, for an excess of chloride does not necessarily mean an excess of $\mathrm{Na}$. I do not believe the aqueous humour is a transudate for the same reasons that $I$ do not think it is a dialysate. 
The importance of Leber's teaching was that it focused attention on the formation of the aqueous humour in the ciliary processes, that it circulated to the anterior chamber, and gained an exit at the canal of Schlemm. The nature of this fluid had to wait until more accurate methods of chemical analyses were available.

Is the aqueous humour a secretion?-In answering this question, Friedenwald and Pierce (1931) have said it all depends on the definition of the word secretion. They have written-"If one defines a secretion as a cellular product which contains some substance not present in the mother liquid or present in marked excess in comparison with the mother liquid, then the aqueous is not a secretion. If, on the other hand, one defines secretion as a substance whose formation is the result of work done by the tissues then the aqueous is a secretion."

I think I would go a little further than Friedenwald and Pierce. On the following grounds I believe the aqueous humour is produced by a process of secretion.

1. There is evidence that work is done or energy is expended in its formation. This must be so or the membrane in the posterior chamber of the eye would not have an irreversible permeability.

2. With regard to its specific secretion, it does not necessarily follow that because a specific substance has not yet been identified it is not present in the aqueous humour and manufactured in some site in the eye. Meyer and Palmer (1936) in fact believe that they have isolated a polysaccharide acid called hyaluronic acid in the aqueous humour, and no such material has been found in the blood. It will be interesting to note if Meyerr and Palmer's work is confirmed.

3. In my investigations I have noted a certain similarity between the eye and a secretory mechanism, namely the stomach-

$i$. The blood-aqueous barrier and the blood-gastric juice membrane appear to hold back to the same extent freely diffusible substances such as urea, sugar, and creatinine.

ii. 50 per cent. glucose injected intravenously does not inhibit gastric secretion, nor does it materially affect the intra-ocular pressure. When I compare the volume of gastric juice secreted with the intra-ocular pressure I am assuming for reasons I have given in my second lecture that a fall in the intra-ocular pressure indicates an inhibition in the formation of the aqueous humour.

iii. 30 per cent. $\mathrm{NaCl}$ inhibits the secretion of gastric juice and also the formation of the aqueous humour. In both the stomach and the eye this inhibition of fluid formation may last several hours after the injection, not, as in the case of dialysates only over the period the injection is being given.

The evidence upon which I believe that the aqueous humour 
is produced by secretion is therefore based more on physiological facts than upon data such as:-

1. Anatomy of the ciliary processes.

2. Cytological evidence of functional activity.

3. Electrical evidence.

4. The stimulation of secretion by drugs or by nerves; and in the opinion of many workers these last four methods of investigation in themselves produce very strong evidence as to the secretory nature of the intra-ocular fluid.

In conclusion I would like to thank the Honorary Staff of Moorfields Royal Ophthalmic Hospital for the honour they have done me in inviting me to give these lectures.

\title{
REFERENCES
}

Friedenwald and Pierce (1931).-Bull. Johns Hopkins Hosp., Vol. XliX, p. 259.

Graham (1876).-T. Graham Chemical and Physiological Researches.

MEYer and PALMER (1936).-Amer. Jl. of Ophthal., Vol. XIX, p. 859.

Robertson (1939).-Brit. Jl. of Ophthal., Vol. XXIII, pp. 106, 170.

Robertson and Williams (1939).-Jl. Physiol., Vol. XCV, p. 139.

\section{A DESCRIPTION OF A MONSTER, DIPROSOPUS TETROPHTHALMUS}

\section{With notes on the histology of the median eyes by John Maude}

\author{
BY

\section{ISADORE BRODSKY*} \\ DEPARTMENT OF PATHOLOGY, UNIVERSITY OF SYDNEY
}

AN uncommon monstrosity which is an example of superior duplication, was recently presented to the Department of Pathology, University of Sydney, by the Royal Alexandra Hospital for Children.

The specimen may be designated diprosopus tetrophthalmus in association with anencephaly.

In the following notes the external features will be described and illustrated; a short description of the internal anatomy, the microscopical appearances of the median eyes, and the radiographical features (together with reproductions of the radiographs) are also incorporated.

External description.-The foetus is a white female. It weighs 2,668 grammes ( 5 pounds, 14 ounces) and the length from the most

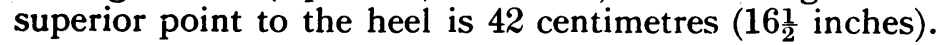

\footnotetext{
* Working under a grant from the National Health and Medical Research Council, 1938.
} 